\title{
Gastrointestinal Infections in IBD Flares: Can PCR-Based Stool Tests Differentiate the Smoke from the Fire?
}

\author{
Jordan E. Axelrad ${ }^{1}$ • Jenny S. Sauk ${ }^{2}$ \\ Published online: 24 March 2020 \\ (c) Springer Science+Business Media, LLC, part of Springer Nature 2020
}

Gastrointestinal (GI) infections are a major cause of morbidity and mortality worldwide [1,2]. In the past, the diagnosis of infective pathogens was accomplished via microscopic examination or culture of, or detection of coproantigens in stool samples. These methods are limited by a lengthy turnaround time, poor sensitivity, a requirement for experts in microscopy, difficulty in isolating non-bacterial agents, and the influence of preanalytical factors such as antibiotics. Using these conventional methods and owing to infrequent detection, non-Clostridioides difficile enteric infections were thought to be minor contributors to the pathogenesis and the disease course of inflammatory bowel disease (IBD) [3, 4]. While multiple studies have supported the involvement of $C$. difficile toward exacerbations of IBD, with a crosssectional prevalence up to $16 \%$ during an acute flare, far less is known about non-C. difficile pathogens [5]. Recently, rapid and highly sensitive stool molecular multiplex polymerase chain reaction (PCR) panel assays, or GI pathogen panels (GPPs) have replaced relatively insensitive culturedependent methods for the detection of enteric pathogens in clinical practice. GPPs enable the simultaneous qualitative detection and identification of nucleic acids from multiple bacteria, viruses, and parasites directly from stool samples in Cary Blair transport medium obtained from individuals with signs and/or symptoms of GI infection. Using this technology, there has been renewed interest in the impact of enteric pathogens on IBD pathogenesis beyond $C$. difficile.

A few recent reports have evaluated stool multiplex PCR panels in patients with IBD [6-8]. In a cross-sectional study

Jordan E. Axelrad

Jordan.Axelrad@nyulangone.org

1 Inflammatory Bowel Disease Center at NYU Langone Health, Division of Gastroenterology and Hepatology, NYU School of Medicine, New York, NY, USA

2 UCLA Center for Inflammatory Bowel Diseases, Vatche and Tamar Manoukian Division of Digestive Diseases, David Geffen School of Medicine At UCLA, Los Angeles, CA, USA using the BioFire ${ }^{\circledR}$ FilmArray ${ }^{\circledR}$ GPP, a platform capable of detecting 22 organisms, including common pathogenic strains of bacteria, parasites, and viruses during symptomatic relapse in 214 patients with IBD, enteric infection was detected in $27 \%$ of tests with $C$. difficile as the most common (13\%) followed by Escherichia coli subtypes (8\%) and viruses (5\%), largely norovirus [5]. IBD therapy escalation was less frequent in those with a positive stool test. In a follow-up analysis of 277 patients with Crohn's disease (CD), 300 patients with ulcerative colitis (UC), and 8826 patients without IBD who underwent a BioFire ${ }^{\circledR}$ FilmArray ${ }^{\circledR}$ GPP for diarrhea, non- $C$. difficile enteric infection was detected in $18 \%, 16 \%$, and $27 \%$ of tests in patients with $\mathrm{CD}, \mathrm{UC}$, and without IBD, respectively [6]. In this study, compared with patients without IBD, CD had a higher prevalence of norovirus and Campylobacter, whereas UC had a lower prevalence of norovirus and a higher prevalence of Campylobacter, Plesiomonas, and E. coli subtypes.

Despite these data, the clinical, laboratory, and endoscopic overlap between GI infection and exacerbation of IBD pose a diagnostic challenge. Several studies have suggested that negative GPP testing is associated with fewer IBDrelated treatment modifications. The consequences of these decisions are unclear. Axelrad et al. reported that enteric infections detected in symptomatic flares did not impact IBD outcomes following testing, but in Ahmad et al. detection of enteric infections via GPP testing was associated with higher hospital-related healthcare utilization, presumably from consequent delay in necessary IBD treatment escalation $[6,8]$. In the setting of $C$. difficile enteric infections, Lukin et al. demonstrated that severe outcomes, including death, sepsis, and/or colectomy, occurred with greater frequency in patients without IBD treatment escalation than in those with therapy escalation [9]. Therefore, given the greater sensitivity of GPP-based testing, understanding the significance of pathogen detection in active IBD and the rates of asymptomatic colonization or nonviable nucleic acids in inactive IBD remains important. During a symptomatic flare, an 
enteric pathogen may be the sole etiology as a clinically significant GI infection, coexist as a complicating active factor or inactive factor as a bystander, or merely be present as an asymptomatic colonizer. As these clinical circumstances require divergent management strategies, it is of utmost importance to determine whether detected GI pathogens are clinically significant or are just colonizers dependent on $\mathrm{CD}$ or UC disease activity.

In this issue of Digestive Diseases and Sciences, Limsrivilai and colleagues compared the cross-sectional prevalence of GI infections as detected by the BioFire ${ }^{\circledR}$ FilmArray ${ }^{\circledR}$ GPP among 333 IBD patients with active and quiescent disease versus 52 healthy controls without IBD [10]. Nearly one-third of active patients tested positive compared with $8 \%$ of those with quiescent disease and $14 \%$ of healthy controls. Limiting active patients to those with symptoms for less than one week yielded a $50 \%$ stool positivity rate. This is the first study to examine quiescent carriage of enteric pathogens in IBD using a GPP. These data confirm that enteric pathogens are more common during acute symptom exacerbations and may be important factors contributing to the disease course, in particular flares. With regard to specific pathogens in active versus quiescent patients, the authors demonstrated that $C$. difficile in UC, and norovirus, enteropathogenic E. coli (EPEC) and $C$. difficile in CD are likely clinically significant based on the differences in prevalence of these organisms between actively inflamed and quiescent patients. Furthermore, the authors show that IBD treatment escalation occurred less frequently in active IBD patients with positive GPP stool tests than in patients with negative GPP stool tests. Though the follow-up period was only 90 days, surgery and hospitalization rates remained similar between those with positive and negative GPP tests. Interestingly, exacerbations complicated by viral infections were subsequently followed by a less aggressive clinical course compared to exacerbations without a pathogen. Major limitations of the present study included its cross-sectional design, the heterogeneity of the active IBD group that included a mixture of acute flares and those without clinical flares but with persistent disease activity, and a small sample size for specific pathogens.

Despite these data further confirming the possible involvement of enteric pathogens in flares of IBD and how the use of these GPPs may assist early diagnosis and management while avoiding unnecessary treatment, many questions remain. Are symptom exacerbations complicated by an enteric pathogen a distinctive subset of patients with IBD flare with a unique impact on the progression of IBD? Do they require specific management in order to optimize outcomes? Do other pathogens, including other viruses, fungi, and bacteriophages not routinely detected by conventional or multiplex molecular techniques, impact IBD pathogenesis? Do GI pathogens increase the risk of incident IBD in patients without IBD? Delineation of the importance of enteric pathogens in flares of IBD will require prospective clinical and molecular data, with specific attention to changes in the gut microbiota and the immune response before, during, and following a GI infection. This is particularly important as an inflamed mucosa may increase the risk of pathogen acquisition, complicating the ascertainment of whether a detected pathogen is a bystander versus a trigger. Management strategies will need to be compared and correlated with clinical and molecular data in order to evaluate whether escalation of IBD therapy in addition to antimicrobial therapy confers an outcome benefit. Undoubtedly, there are many unresolved issues surrounding GI pathogens in the IBD population that should prompt future studies.

\section{References}

1. Riddle MS, DuPont HL, Connor BA. ACG clinical guideline: diagnosis, treatment, and prevention of acute diarrheal infections in adults. Am J Gastroenterol. 2016;111:602-622.

2. Scallan E, Hoekstra RM, Angulo FJ, et al. Foodborne illness acquired in the United States-major pathogens. Emerg Infect Dis. 2011;17:7-15.

3. Meyer AM, Ramzan NN, Loftus EV, et al. The diagnostic yield of stool pathogen studies during relapses of inflammatory bowel disease. J Clin Gastroenterol. 2004;38:772-775.

4. Mylonaki M, Langmead L, Pantes A, et al. Enteric infection in relapse of inflammatory bowel disease: importance of microbiological examination of stool. Eur J Gastroenterol Hepatol. 2004; 16:775-778.

5. Axelrad JE, Joelson A, Nobel YR, et al. Enteric infection in relapse of inflammatory bowel disease: the utility of stool microbial PCR testing. Inflamm Bowel Dis. 2017;23:1034-1039.

6. Axelrad JE, Joelson A, Green PHR, et al. Enteric infections are common in patients with flares of inflammatory bowel disease. Am J Gastroenterol. 2018;113:1530-1539.

7. Axelrad JE, Freedberg DE, Whittier S, et al. Impact of gastrointestinal panel implementation on health care utilization and outcomes. J Clin Microbiol. 2019;57:e01775.

8. Ahmad W, Nguyen NH, Boland BS, et al. Comparison of multiplex gastrointestinal pathogen panel and conventional stool testing for evaluation of diarrhea in patients with inflammatory bowel diseases. Dig Dis Sci. 2019;64:382-390. https://doi.org/10.1007/ s10620-018-5330-y.

9. Lukin DJ, Lawlor G, Hudesman DP, et al. Escalation of Immunosuppressive therapy for inflammatory bowel disease is not associated with adverse outcomes after infection with clostridium difficile. Inflamm Bowel Dis. 2018;25:775-781.

10. Limsrivilai J, Saleh ZM, Johnson LA, et al. Prevalence and effect of intestinal infections detected by a PCR-based stool test in patients with inflammatory bowel disease. Dig Dis Sci. (Epub ahead of print). https://doi.org/10.1007/s10620-020-06071-2.

Publisher's Note Springer Nature remains neutral with regard to jurisdictional claims in published maps and institutional affiliations. 